\title{
Die wepsische Sprache mit neuen Mitteln dargestellt
}

RIHo GrünthaL: Vepsän kielioppi [Grammatik des Wepsischen]. Hilfsmittel für das Studium der finnisch-ugrischen Sprachen XVII. Suomalais-Ugrilainen Seura. Helsinki 2015. 332 Seiten + Sprachproben, insgesamt 347 Seiten.

Die wepsische Sprache ist von ihrem Verbreitungsgebiet her die östlichste bekannte und dokumentierte ostseefinnische Sprachform. Sie wird nur noch von weniger als 3000 Menschen gesprochen und ist eine der bedrohten ostseefinnischen Sprachen. Das Wepsische wurde seit dem 19. Jahrhundert erforscht. Eine besondere Erwähnung verdient die Tatsache, dass bereits in der ersten Hälfte des 20. Jahrhunderts eine ausgesprochen umfangreiche und gründliche syntaktische Untersuchung zum Wepsischen vorgelegt wurde. Das Werk Vepsän murteiden lauseopillinen tutkimus [Syntaktische Untersuchung der wepsischen Dialekte] von Lauri Kettunen zählt vom Ansatz her zu den letzten, die sich an dem Muster der syntaktischen Untersuchungen zu den finnischen Dialekten orientierten, die der Kreis um Setälä Ende des 19. Jahrhunderts verfasste. Sein Umfang hebt es jedoch aus den Untersuchungen über die ostseefinnischen Sprachen hervor. Mit Ausnahme des Finnischen und des Estnischen wurde über keine andere ostseefinnische Sprache eine entsprechend umfangreiche syntaktische Darstellung veröffentlicht. Die nun vorliegende Grammatik ist nach Kettunens Untersuchung die erste in Finnland und auf Finnisch verfasste, umfassendere Darstellung der wepsischen Sprache.

Der Verfasser nennt als seine Zielsetzung, ein Gesamtbild der wepsischen Sprache und ihrer grammatischen Struktur zu geben, und äußert die Hoffnung, das Buch könne Lesern, die mit den sprachwissenschaftlichen Termini vertraut sind, als Sprachführer dienen, wenngleich es an sich nicht als Lehrbuch konzipiert ist. Es enthält acht Kapitel, Quellen- und Literaturverzeichnisse sowie Sprachproben. Die Kapitel sind: 1. Einleitung, 2. Die Wepsen und die wepsische Sprachgemeinschaft, 3. Lautsystem, 4. Morphologie und Flexion, 5. Satzbildung, 6. Dynamik der der Satzgefüge und Semantik, 7. Pronomina, Deixis und Partikeln sowie 8. Komplexe Sätze. Diese Kapitel gliedern sich dem Ver- 
fasser zufolge in solche, in denen strukturelle Einzelheiten betrachtet werden, und solche, in denen die wichtigsten Themen behandelt werden, die aus der von Bedeutungen und Wörtern, Flexionssuffixen und Sätzen gebildeten Gesamtheit hervorgehen. Neben den genannten Hauptkapiteln gibt es insgesamt 37 Unterkapitel sowie fünf Unterkapitel von Unterkapiteln.

Im einleitenden Kapitel 1 werden die heutige Situation, die Dialekte und die Stellung des Wepsischen aus der Perspektive der ostseefinnischen Sprachen und der arealen Sprachumgebung Kompakt und effizient dargestellt. Die der Einleitung beigefügte Karte des wepsischen Sprachgebiets zeigt in schonungsloser Deutlichkeit, was mit dem Wepsischen geschehen ist und geschieht. In den Unterkapiteln werden das verwendete Material, der Aufbau des Buches sowie die Schreibweise der Beispiele und Sprachproben vorgestellt.

Als zentrales Material wurde die oben erwähnte syntaktische Untersuchung von Kettunen verwendet. Der Verfasser begründet diese Entscheidung mit der Repräsentativität von Kettunens Material und damit, dass die Bedeutung eigenen Materials im Verhältnis zum Zeitaufwand für die Sammeltätigkeit geringfügig gewesen wäre. Die Entscheidung wurde auch dadurch beeinflusst, dass der Verfasser das gesprochene Wepsisch darstellen will, wofür Kettunens Materal eine breite Basis bietet.

Der überwiegende Teil des Materials wurde also einer anderen Untersuchung entnommen, was die ungeheure Arbeitsleistung des Verfassers jedoch nicht schmälert. Den in Kapitel 1.3 angeführten Abkürzungen für die Sprachproben zufolge enthält das Buch die beachtliche Zahl von 1879 Beispielen. Aus Kettunens syntaktischer Untersuchung stammen davon 1258 Beispiele, die der Verfasser neu behandelt und vereinfacht hat. Die restlichen 621 Beispiele, ebenfalls eine große Menge, wurden früher veröffentlichten Sprachproben und anderen Korpora entnommen. Dabei war größtenteils eine vollständige Neubearbeitung erforderlich. Rein rechnerisch entfallen auf jede Seite 5,6 Beispiele. Dennoch ist das Buch splendid gesetzt, und die Menge der Beispiele beeinträchtigt die Lektüre nicht.

Einige Überschriften stimmen nicht mit dem Inhalt überein. Zum Beispiel werden im Kapitel 1.3 unter der Überschrift Die Schreibweise der Beispiele und Sprachproben tatsächlich die für die Sprachproben verwendeten Abkürzungen erläutert. Hinsichtlich der Schreibweise der Beispiele und Sprachproben wird lediglich auf das Kapitel 3.3 
Die Prinzipien der Vereinfachung der Schreibweise verwiesen. Der Inhalt des Kapitels 3.3, das fünf Seiten umfasst, hätte durchaus in Kapitel 1.3 Platz gefunden, was das Problem jedoch nicht gelöst hätte. In diesem Kapitel werden in geeigneten Teilen die auf der Schriftsprache basierende Schreibweise und die Vereinfachung dargestellt. Die Schreibweise der Schriftsprache selbst oder auch nur ihre Grapheme werden jedoch kaum vorgestellt. Um sich über letztere $\mathrm{zu}$ informieren, muss der Leser das Kapitel 3.1 über das Phonemparadigma zu Rate ziehen. Alles in allem ist das Kapitel 1.3 überflüssig. Wenn der Verfasser die Abkürzungen für die Sprachproben in das Kapitel 1.1 integriert und den Rest gestrichen hätte, bliebe dem Leser unnötige Arbeit erspart.

Kapitel 2 ist im Wesentlichen ein wissenschaftsgeschichtlicher Überblick über die wepsische Sprache und ihre Sprecher. Es enthält eine angemessene Menge an Informationen, die zwar nichts mit der Grammatik zu tun haben, aber für angehende Erforscher des Wepsischen notwendig sind. Die in den Unterkapiteln gegebenen Informationen über das in frühgeschichtlichen schriftlichen Quellen begegnende Volk ves', über die Geschichte der Erforschung des Wepsischen und die daran beteiligten Wissenschaftler, über die
Veränderungen in der Sprecherzahl und den Verbreitungsgebieten des Wepsischen, über die typischen Besonderheiten des Wepsischen im Vergleich $\mathrm{zu}$ den anderen ostseefinnischen Sprachen und über die Bezeichnungen für das Wepsische und die Wepsen unter dem Aspekt der Identifikation und der sprachlichen Definition bieten eine kompakte Lektüre von hoher Qualität. Das Kapitel 2.6 über die Bezeichnungen für das Wepsische und die Wepsen hebt sich durch seine forschungsorientierte Herangehensweise von den anderen Unterkapiteln ab. Dies erklärt sich aus der wissenschaftlichen Tätigkeit des Verfassers: Er hat über die hier behandelten Themen drei Untersuchungen verfasst. Dennoch hätte man dieses Kapitel den anderen Unterkapiteln angleichen und es kürzen können, indem man zumindest auf die Beispiele verzichtet hätte, die hauptsächlich eine schmückende Funktion haben.

In Kapitel 3 wird das Lautsystem des Wepsischen vorgestellt. Die Unterkapitel befassen sich mit den in der Schriftsprache verwendeten Zeichen sowie mit den Faktoren, die sich auf Qualität und Dauer der Laute auswirken, nämlich Assimilation und morphophonologischer Wechsel. Die Themen werden präzise dargestellt, doch die Darstellung wäre anschaulicher ausge- 
fallen, wenn anstelle der Beispiele eine Tabelle der Flexionstypen verwendet worden wäre, wie man sie zum Beispiel in der grammatischen Darstellung des Livischen findet (s. Viitso 2008: 346-353).

In Kapitel 4 ist die Morphologie an der Reihe. Es handelt sich um das umfangreichste Kapitel mit 115 Seiten, drei Unterkapiteln und acht Unterkapiteln zweiter Ordnung. Vorgestellt werden das wepsische Morphemsystem, das sich von demjenigen des Finnischen nur im Hinblick auf die spät entstandenen Kasusformen und die Reihenfolge der Possessivsuffixe unterscheidet, die Kasusformen der Nomina (vor allem des Mittelwepsischen), d.h. der Substantive und Adjektive, nach den Stammtypen und ihre Verwendung, der Numerus, die Possessivsuffixe und ihre Verwendung, Denominalisierung und Komposita. Nach den Nomina werden die die Konjugation der Verben, die Infinitive und Partizipien und ihre Verwendung sowie die Deverbalisierung dargestellt.

In diesem Kapitel zeigt sich, dass Grünthals Grammatik trotz der eingangs erwähnten neuen Darstellungsweisen nicht von der Tradition der finnougristischen Grammatiken abweicht; im Zusammenhang mit den Kasusformen werden auch ihre Verwendung und ihre Funktionen, d. h. Fragen der Syntax, behan- delt. Diese Praxis dürfte ursprünglich entstanden sein, weil die Syntax der bedrohten uralischen Sprachen kaum erforscht war und man am besten die mit der historischen Morphologie Hand in Hand gehende Kasussyntax kannte, die im Zusammenhang mit der Beschreibung der Kasusformen dargestellt werden konnte. Die Situation ist völlig anders als in der Tradition der Grammatik des Lateinischen und Griechischen. Dort war es üblich, Morphologie und Syntax separat zu behandeln. Die Tradition wirkt sich nicht eigentlich störend aus, zumindest nicht in diesem Kapitel, aber gerade im Fall des Wepsischen ist die Lösung ungeschickt, da es bereits eine umfangreiche syntaktische Darstellung gibt. In einem Kapitel über die Morphologie syntaktische Funktionen wie Subjekt und Prädikat $\mathrm{zu}$ behandeln, erscheint nach heutigem Maßstab zweifellos verworren und altmodisch.

Es wäre sinnvoll gewesen, größeres Gewicht auf die Morphologie zu legen, da der größte Teil der syntaktischen Informationen sich auch in Kettunens Untersuchung findet. Trotz des relativ großen Umfangs liefert das Kapitel nur knappe Informationen. Beispielsweise wird das im Lokalkasussystem des Wepsischen wichtige Suffix -päi, das dem finnischen Wort päin entspricht, nicht separat erwähnt. Der 
Verfasser gibt sowohl in den Tabellen 4-1-4-3 als auch bei den Beispielen für Elativ und Ablativ (S. 77-78; 85-86) zu verstehen, dass sich das Suffix -päi in den Woher-Kasus eingebürgert hat. Dieses Suffix hat sich als Teil des Woher-Kasus beim Elativ und Ablativ sowie bei funktional dem Woher-Kasus entsprechenden Adverbien eingebürgert, aber nur dann, wenn es sich um lokale oder temporale Bedeutung handelt. Beim habitiven Ablativ und manchmal auch beim Elativ kann das Suffix entfallen. Dies ist beim Ablativ in schriftsprachlichen Texten ziemlich regelmäßig der Fall, z. B. -- ot'iba hänel sobiit kahthesadha nel'hakümnhe rubl'ha-(SUST 70: 31); vgl. ol'oša ot'i generaloupäi šenrl'in pälpäi -- (SUST 70: 84); -- otiba hänel kalun i löiba sil händast pähä (Uz, Mat. 27:30); Epafras, kenes tö olet kulunuded, -- (Uz Kol. 1:7). Statt aufgrund des betreffenden Suffixes eine unnötige Trennung zwischen dem Illativ und der lativischen Kasusform -hVpäi vorzunehmen, wäre eine umfassendere Darstellung des Suffixes wünschenswert gewesen, wie sie sich in früheren Untersuchungen findet (s. Kettunen 1943: 279; 331; 366-368; Zaitseva 1981: 186-189).

Kapitel 5 über die Satzbildung beginnt mit der Wortstellung; darauf folgen die Kongruenz (des Subjekts und der anderen Funktio- nen), der Prädikativsatz, der verneinende Satz und die Vielförmigkeit der Negation, das Bestimmungsglied, die adpositionale Phrase, die Komparation, der Fragesatz, die Adverbien und die Numeralia. Bei einem Teil der Untertitel ist schwer zu verstehen, dass es sich um Unterkapitel zur Satzbildung handelt. Zudem werden aufgrund der schlechten Kapiteleinteilung teilweise dieselben Themen wiederholt. Mit der Lektüe dieses Kapitels kann man nicht beginnen, bevor man sich mit der Verwendung der Kasusformen in Kapitel 4 bekannt genmacht hat, wo die Funktionen samt Kasusformen erklärt wird.

Der Untertitel Prädikativsatz ist ein Beispiel für die Ungeschicklichkeiten, die auf die eigenwillige Entscheidung des Verfassers zurückgehen. Der Begriff wird sowohl als übergeordnete Kategorie verwendet, die dem allgemein gebräuchlichen Terminus Nominalsatz (oder Kopulasatz) entspricht, wie auch als Unterkategorie, die dem in der Fennistik bekannten Prädikativsatz entspricht. Daraus resultiert, dass als Untertypen des Prädikativsatzes der äquative Prädikativsatz, der attributive Prädikativsatz, der Possessivsatz, der Lokativsatz und der Existenzialsatz auftreten. Da das vorliegende Werk auf Finnisch geschrieben ist, wäre es wünschenswert gewesen, die fin- 
nischen Termini der allgemeinen Praxis entsprechend zu verwenden. Infolge der Verwendung eines eigenwilligen Terminus stellt sich bei einigen Beispielen die Frage, ob sie nur falsch gewählt wurden oder ob der Verfasser mit der fennistischen Grammatiktradition mangelhaft vertraut ist. Auf den Seiten 185 und 186 werden ein lokativischer Satz, der den Standort des Subjektdesignats angibt, und ein Existenzialsatz, der die allgemeine Existenz ausdrückt, vorgestellt. Nach Ansicht des Rezensenten handelt es sich bei beiden Beispielen um Existenzialsätze.

Kapitel 6 behandelt Transitivität, Impersonalia, modale Konstruktionen, Agens und Patiens sowie Topik. In diesem Kapitel fühlt man sich erneut eher an eine Untersuchung als an eine Darstellung der Grammatik erinnert. Auch in einer deskriptiven Grammatik wäre es nicht nötig, einen Sachverhalt zu belegen, als würde der Leser ihn andernfalls nicht glauben. Es würde sicher genügen, das System und die Abweichung darzustellen. In diesem Kapitel sind auch die Erläuterungen recht verworren. Im Zusammenhang mit Agens und Patiens heißt es: „Ein in subjektartiger Position stehendes Satzglied wird auch im Wepsischen anders markiert als ein normales Subjekt, also Verursacher oder Ausführer einer Handlung“. Was ist eine subjektartige Position? Hätte man nicht einfacher von Agensadverbial oder Subjekt des Partizips sprechen können?

Kapitel 7 beginnt mit einer Deklinationstabelle der Personalpronomina, doch bei den anschließend dargestellten Demonstrativ- und Indefinitpronomina wird auf eine Deklinationstabelle verzichtet und direkt auf ihre syntaktischen Eigenheiten eingegangen. Das Kapitel endet mit den enklitischen Partikeln. Die Hauptüberschrift des Kapitels steht mit den Überschriften der Unterkapitel in Übereinstimmung. Dennoch ist schwer zu verstehen, warum sie gerade in diesem Kapitel dargestellt werden. Gehört die Deklinationstabelle der Personalpronomina nicht zur Morphologie? Warum werden für die anderen Pronomina keine Deklinationstabellen gegeben? Es entsteht zwangsläufig der Eindruck, das die Deklinationstabelle eingesetzt wurde, um das Unterkapitel über die Personalpronomina zu verlängern, das andernfalls sehr kurz ausgefallen wäre.

Im letzten, 8. Kapitel werden komplexe Sätze, d.h. die Arten der Koordination oder Subordination der Teilsätze dargestellt. Das letzte Unterkapitel behandelt allerdings Verbverbindungen, die in keiner Weise mit der Überschrift des Hauptkapitels in Einklang stehen. 
Dem Bucheinband verleihen Bilder von einem Blockhaus eine stille Schönheit, und der Text ist angenehm splendid gesetzt. Insgesamt hinterlässt die Lektüre einen vorwiegend positiven Eindruck, trotz der stellenweise begegnenden unbeholfenen Lösungen. Erwähnenswert ist auch, dass 18 der 49 Unterkapitel mit einem eigenen Literaturverzeichnis versehen wurden. Die Sprachproben am Ende des Bandes bieten dem Leser die Möglichkeit, die verschiedenen Sprachformen des Wepsischen kennenzulernen, ohne zu anderen Büchern greifen zu müssen.

Vom Aufbau her weicht das Werk in einigen Teilen recht stark von den üblichen Grammatiken ab und weist mehrere ausgesprochen eigenwillige Lösungen auf. Da es keinerlei Register enthält, kann man es nicht als Nachschlagewerk verwenden, ohne es einmal durchzulesen und die wichtigen Stellen selbst $\mathrm{zu}$ markieren. Dies ist ein bedauerlicher Mangel, der sich unmittelbar auf die Nutzbarkeit des Werks auswirkt. Der Verfasser hätte durchaus die Möglichkeit gehabt, ein recht umfassendes Register zu erstellen. Das Werk enthält nämlich außer den im Inhaltsverzeichnis genannten Haupt- und Unterkapiteln 87 unnummerierte Untertitel. Beispielsweise finden sich in Kapitel 4.3.1 über die Flexionskategorien
14 unnummerierte Untertitel, die teilweise in einer hierarchischen Beziehung zueinander stehen (z. B. Modi und Imperativ, Konditional usw.). Auf Seite 284 wird bei den unnummerierten Untertiteln Relativpronomina und Interrogativpronomina lediglich auf andere Kapitel verwiesen. Als Untertitel sind sie also völlig überflüssig, aber gerade auf diese Weise hätte ein Register erstellt werden können. Die unnummerierten Untertitel wirken visuell auflockernd. Andererseits ist es schwierig, sie später zu nutzen, da das Buch kein Register aufweist und das Inhaltsverzeichnis sie nicht anführt. Um die unnummerierten Untertitel nutzen zu können, muss der Leser also selbst die entsprechenden Seitenzahlen notieren oder sich auf sein $\mathrm{Ge}$ dächtnis verlassen. Die inkohärente Planung des Aufbaus zeigt sich darin, dass ein Teil der Überschriften von Unterkapiteln mit zusätzlichen Informationen ergänzt und erneut verwendet werden musste (z. B. Imperativ und Imperativ der Reflexivkonjugation). In das Register hätte auch ein Teil der fettgedruckten Wörter aufgenommen werden können, deren Gesamtzahl 188 beträgt. In der Zeit des Internet könnte das Register nachträglich dort veröffentlicht werden. Wenn man die erwähnten unnummerierten Untertitel und fettgedruckten Wörter 
nutzt, dürfte die Erstellung des Registers keine große Mühe bereiten.

Neben dem Fehlen des Registers fällt eine häufig eigenwillige und bisweilen willkürliche Herangehensweise auf, die teilweise bereits erwähnt wurde. Die Tabellen gehen mal vom Mittelwepsischen, mal von der Schriftsprache aus. Erklärungen für die jeweilige Wahl der Sprachform werden nicht gegeben. Der Leser kann nur vermuten, dass das Mittelwepsische wegen der Menge der Beispiele gewählt wurde; von 1879 Beispielen stammen 1001 aus dem Mittel- und Nordwepsischen. Diejenigen, die durch das vorliegende Werk erstmals mit dem Wepsischen in Berührung kommen, könnten jedoch zu der irrtümlichen Auffassung gelangen, dass der mittelwepsische Dialekt gewählt wurde, weil er der Schriftsprache am nächsten stünde. So verhält es sich jedoch nicht. Beispielsweise sind in der Deklinationstabelle der Personalpronomina im Singular auf Seite 275 die Allativ- und Adessivformen der 1. und 2. Person identisch. Dieser Synkretismus ist eine Besonderheit des Mittel- und Nordwepsischen (s. Kettunen 1943: 382-383), doch auch hierzu wird keinerlei Erklärung gegeben. Generell sind die Informationen über die Dialekte spärlicher als in früheren Untersuchungen und Grammatiken, was einen deutlichen Rückschritt darstellt.
Auch darstellungstechnische Inkonsequenzen finden sich im ganzen Werk. Bei den Tabellen 4-1-4-3 über die Deklination der Nomina im Mittelwepsischen werden nur in der Tabelle 4-2 die Morpheme mit einem Strich abgetrennt. Auf den Seiten 98-102 ist nur das komitativische -ne fettgedruckt, nicht aber die anderen Kasusendungen -či, -li, - $h V p a ̈ i,-i n$. Auf Seite 100 werden bei dem lativischen Suffix - hVpäi die Vokale der Allomorphe durch Verwendung des Symbols V berücksichtigt, während bei der Darstellung des Illativs auf Seite 72 nur die Endung -he erwähnt wird. Das Suffix - ze wird an keiner der beiden Stellen erklärt. Auch die Vereinheitlichung der Bildung von Untertiteln hätte den Gesamteindruck klarer erscheinen lassen (z. B. Tempus, aber Modi). Ein Teil dieser Inkonsequenzen dürfte auf fehlendes Korrekturlesen zurückzuführen sein. Bei den Quellenangaben zu den Beispielen steht in zehn Fällen MSFOu, bei den restlichen dagegen SUST. Hinzu kommen Markierungen, die der Verfasser offenbar für den eigenen Gebrauch eingefügt hat (z. B. POSS) und fehlerhafte Quellenangaben wie SUST 79 und SUST 120.

Selbst wenn alle oben erwähnten, vor allem auf technischer Inkonsequenz beruhenden Mängel sowie das Fehlen eines Registers 
bei der nächsten Auflage behoben und die Benutzerfreundlichkeit des Werks verbessert würden, bleibt noch ein Stein im Schuh. Dem Unterzeichneten blieb bis zum Schluss unklar, an wen sich diese Grammatik richtet. So ist es, obwohl die Lektüre, wie oben gesagt, an sich einen positiven Eindruck hinterlässt. Für Wissenschaftler, die die Untersuchung von Kettunen und die sonstige bisherige Forschung kennen, bietet das vorliegende Werk keinen Ersatz, da es schlicht und einfach weniger Informationen enthält. Es sei daran erinnert, dass Sprachwissenschaftler fähig sind, die gesuchten Informationen unabhängig von Begriffen und Darstellungsweisen $\mathrm{zu}$ finden, sofern sie nur auf irgendeine Weise präsentiert werden. Werden sie nicht vorgelegt, können die so entstehenden Lücken weder durch moderne Begriffe noch durch eine neue Darstellungsweise aufgewogen werden. Für Neulinge im Bereich der Erforschung des Wepsischen bietet das Werk gute Basisinformationen, aber auch ihnen kann es wohl nicht als vollständige Grammatik dienen, da zu vielen Punkten folgerichtig dargestellte Angaben fehlen. Sofern das Werk zusammen mit anderen Quellen genutzt werden soll, verringert sich seine Bedeutung als Grammatik wesentlich. In der jetzigen Fassung lässt sich das Werk nur erschöpfend nutzen, indem man es als sachbuchartige Quelle liest. Aus dieser Perspektive betrachtet, ist es ausgesprochen beeindruckend. Es bietet vielfältige Informationen über die wepsische Sprache, die man sich auf Finnisch aneignen kann. Es wird zweifellos das erste Tor werden, durch das der Weg von Neulingen im Bereich der wepsischen Sprachforschung führt.

\section{Nobufumi Inaba}

\section{Literatur}

SUST 70 = Kettunen, Lauri - Siro, Paavo 1935: Näytteitä vepsän murteista [Proben aus den wepsischen Dialekten]. Suomalais-Ugrilaisen Seuran Toimituksia 70. Suomalais-Ugrilainen Seura. Helsinki.

$\mathrm{UZ}=U Z^{\prime}$ Zavet. Uz' Zavet vepsän kielel [Das Neue Testament in wepsischer Sprache]. Biblijan kändmižen institut. Helsinki 2006.

Kettunen, Lauri 1943: Vepsän murteiden lauseopillinen tutkimus [Syntaktische Untersuchung der wepsischen Dialekte]. Suomalais-Ugrilaisen Seuran Toimituksia 86. Helsinki.

Virtso, Tirt-Rein 2008: Liivi keel ja läänemeresoome keelemaastikud [Die livische Sprache und die ostseefinnischen Sprachgebiete]. Eesti Keele Sihtasutus. Tartu-Tallin.

Zaitseva 1981 = Зайцева, М. И 1981: Грамматика вепсского языка (фонетика и морбология) [Grammatik der wepsischen Sprache (Phonetik und Morphologie)]. Наука. Ленинград. 